\title{
Computer Assisted Coronary Intervention by Use of On-line 3D Reconstruction and Optimal View Strategy
}

\author{
S.-Y. James Chen and John D. Carroll \\ Cardiology Division, Department of Medicine \\ University of Colorado Health Sciences Center \\ james@stbernard.uhcolorado.edu, jcarroll@rigel.uhcolorado.edu
}

\begin{abstract}
A novel method has been developed for on-line reconstruction of the 3D coronary arterial tree based on a pair of routine angiograms acquired from any two arbitrary viewing angles using a single-plane or biplane imaging system. An arterial segment of interest (e.g., coronary stenosis) is selected on the projection of reconstructed 3D coronary model. Afterwards, the process of optimal view strategy is employed resulting in foreshortening, overlap, and composite maps relative to the selected arterial segment by which any computer-generated projection associated with the gantry orientation can be previewed. By use of the three maps, the views with minimal foreshortening and vessel overlap for the selected arterial segment of interest can be determined to guide subsequent angiogram acquisitions for interventional procedure. More than 200 cases of coronary arterial systems have been reconstructed. A validation confirmed the accuracy of $3 \mathrm{D}$ length measurement to within RMS $3.1 \%$ error using 8 pairs of angiograms of intra-coronary catheter wire of $105 \mathrm{~mm}$ length.
\end{abstract}

\section{Introduction}

Many endeavors of computer assisted techniques for estimation of the threedimensional (3D) coronary arteries from biplane projection data have been reported [1]-[7]. These methods were based on the known or standard x-ray geometry of projections, placement of landmarks, or the pre-determined vessel shape and on iterative identification of matching structures in two or more views. Other knowledge-based or rule-based systems have been proposed for $3 \mathrm{D}$ reconstruction of coronary arteries by use of of a vascular network model [8]-[12]. Because the rules or knowledge base were organized for certain specific conditions, it is not likely to generalize the $3 \mathrm{D}$ reconstruction process to arbitrary projection data. In [13], the 3D coronary arteries were reconstructed from a set of $\mathrm{x}$-ray perspective projections by use of an algorithm from computed tomography. Due to the motion of heart, only a limited number of projections can be acquired. Therefore, accurate reconstruction and quantitative measurement are not easily achieved. 
The linear approach of closed-form solution for the general 3D reconstruction problem based on 2D projections was a significant development in [14]-[19]. Unfortunately, actual data are always corrupted by noise or errors and the linear approach based techniques may not be sufficiently accurate from noisy data. Hence, optimal estimation has been explicitly investigated. In [20]-[25], a twostep approach was proposed for an optimal estimation of 3D object structures based on maximum-likelihood and minimum-variance estimation. Preliminary estimates computed by the linear algorithm were used as initial estimates for the process of optimal estimation.

In this paper, an on-line 3D reconstruction technique is proposed to accurately reconstruct the coronary arterial trees based on two views acquired from routine angiograms at arbitrary orientation using a single-plane or biplane imaging system. The reconstructed patient-specific 3D coronary arterial tree is used to facilitate the determination of optimal view strategy.

\section{On-line 3D Reconstruction of Coronary Arterial Tree}

After routine cardiac catheterization is initiated, a standard coronary arteriogram is completed in two standard views: one injection in a biplae system and two injections in a single-plane system. With the acquired two angiogram sequences, a pair of images were chosen corresponding to the same or close acquisition time instance (e.g., end-diastole or end-systole) in the cardiac cycles for $3 \mathrm{D}$ reconstruction process. The proposed method of on-line 3D reconstruction consists of three major steps: (1) identification of 2D coronary arterial trees and feature extractions, (2) determination of transformation defining the spatial relationship of the acquired two views, and (3) calculation of 3D coronary arterial structures.

\subsection{Recognition of $2 \mathrm{D}$ coronary arteries}

A semi-automatic system [24] based on the technique of deformation model and segmentation technique was employed for identification of the 2D coronary arterial tree in the given angiograms. The identified centerlines and the branching relationships are used for construction of the vessel hierarchy in each angiogram by their labeling according to the appropriate anatomy and connectivity among the primary and secondary coronary arteries. The labeling process on the coronary tree is performed automatically by traversing the identified vessel centerlines and associated bifurcations, and finally results in a vessel hierarchically directed graph (digraph) containing a set of nodes with corresponding depth levels and two types of arcs (descendant and sibling arcs) defining the coronary anatomy. In the hierarchical digraph, each node represents a vessel in the coronary arterial tree, and the descendent arc connects the nodes between the vessel and its branch. If a vessel has multiple branches, the nodes associated with these branches are connected as a linked list by sibling arcs according to the branching locations relative to the ascendant vessel. With the constructed hierarchical digraph, bifurcation points, directional vectors, and diameters are then calculated 
for all of the coronary arterial bifurcations, and the results are saved into the nodes associated branching vessels in the hierarchical digraph.

\subsection{Determination of transformation defining the spatial relationship of two views}

The spatial relationship between the employed two views can be characterized by a transformation in the forms of a rotation matrix $\boldsymbol{R}$ and a translation vector $\boldsymbol{t}$ with the $\mathrm{x}$-ray source (or focal spot) served as the origin of 3D coordinate space. The geometrical relationship between the two views can be characterized by $\left[x_{i}^{\prime}, y_{i}^{\prime}, z_{i}^{\prime}\right]^{T}=\boldsymbol{R} \cdot\left(\left[x_{i}, y_{i}, z_{i}\right]^{T}-t\right)$. In the first view, let $\left(u_{i}, v_{i}\right)$ denote the image coordinates of the $i$-th object point, located at position $\left(x_{i}, y_{i}, z_{i}\right)$. We have $u_{i}=D x_{i} / z_{i}, v_{i}=D y_{i} / z_{i}$, where $D$ is the perpendicular distance between the $\mathrm{x}$-ray focal spot and the image plane. Let $\left(\xi_{i}, \eta_{i}\right)$ denote the scaled image coordinates, defined as $\xi_{i}=u_{i} / D=x_{i} / z_{i}, \eta_{i}=v_{i} / D=y_{i} / z_{i}$. The second projection view of the employed single-plane imaging system can be described in terms of a second pair of image and object coordinate systems $u^{\prime} v^{\prime}$ and $x^{\prime} y^{\prime} z^{\prime}$ defined in an analogous manner. Scaled image coordinates $\left(\xi_{i}^{\prime}, \eta_{i}^{\prime}\right)$ in the second view for the $i$-th object point at position $\left(x_{i}^{\prime}, y_{i}^{\prime}, z_{i}^{\prime}\right)$ are given by $\xi_{i}^{\prime}=u_{i}^{\prime} / D^{\prime}=$ $x_{i}^{\prime} / z_{i}^{\prime}, \eta_{i}^{\prime}=v_{i}^{\prime} / D^{\prime}=y_{i}^{\prime} / z_{i}^{\prime}$.

In our previously proposed method [24][25], the transformation was calculated based on the identified bifurcation points of the 2D coronary arterial trees in the two views and can be only effective to the images acquired from the biplane systems (or a single-plane system without table movement) during the acquisition. In this paper, a new $3 \mathrm{D}$ reconstruction technique is presented by incorporation of the bifurcation points and the vessel directional vectors of bifurcations. This new technique can be utilized adequately in both single-plane and biplane environments. The proposed method can also be employed to obtain accurate results of $3 \mathrm{D}$ reconstruction even when the table is moving during the injection or with the ECG-triggered mode disabled in acquisitions. For 3D reconstruction, the required prior information (i.e., the intrinsic parameters of each single-plane imaging system) include: (1) the distance between each focal spot and its image plane, SID (focal-spot to imaging-plane distance), (2) the pixel size, $p_{\text {size }}(e . g ., .3 \mathrm{~mm} / \mathrm{pixel})$, (3) the distance $\overline{f f^{\prime}}$ between the two focal spots or the known 3D distance between two points in the projection images, and (4) iso-center distance (with respect to which the rotary motion of the gantry arm rotates) relative to the focal spot.

To calculate the transformation, the objective function was defined by minimization of (1) image point errors in term of the square of Euclidean distance between the 2D input data and the projection of calculated 3D data points and (2) directional vector errors between the $2 \mathrm{D}$ vessel directional vectors and the projection of calculated 3D vessel directional vectors. Given the set of $2 \mathrm{D}$ bifurcation points and directional vectors extracted from the pair of images, an "optimal" estimates of the transformation and 3D bifurcation points were obtained by minimizing: 


$$
\begin{gathered}
\min _{R, \boldsymbol{t}, \hat{\boldsymbol{p}}^{\prime}, v^{\prime}} \mathcal{F}\left(\boldsymbol{R}, \boldsymbol{t}, \hat{\boldsymbol{P}}^{\prime}, \hat{\boldsymbol{v}}^{\prime}\right)=\sum_{i=1}^{n}\left(\hat{\xi}_{i}^{\prime}-\frac{\hat{x}_{i}^{\prime}}{\hat{z}_{i}^{\prime}}\right)^{2}+\left(\hat{\eta}_{i}^{\prime}-\frac{\hat{y}_{i}^{\prime}}{\hat{z}_{i}^{\prime}}\right)^{2}+\left(\hat{\xi}_{i}-\frac{\boldsymbol{c}_{\boldsymbol{1}} \cdot \boldsymbol{p}_{i}^{\prime}+t_{x}}{\boldsymbol{c}_{\boldsymbol{3}} \cdot \boldsymbol{p}^{\prime}{ }_{i}+t_{z}}\right)^{2} \\
+\left(\hat{\eta}_{i}-\frac{\boldsymbol{c}_{\boldsymbol{2}} \cdot \hat{\boldsymbol{p}}_{i}^{\prime}+t_{y}}{\boldsymbol{c}_{\boldsymbol{3}} \cdot \hat{\boldsymbol{p}}^{\prime \prime}{ }_{i}+t_{z}}\right)^{2}+\left\|\hat{\boldsymbol{\nu}}_{i}-\left[\boldsymbol{R}^{-1} \cdot \hat{\boldsymbol{v}}_{i}^{\prime}\right]_{x y}\right\|^{2}+\left\|\hat{\boldsymbol{\nu}}_{i}^{\prime}-\left[\hat{\boldsymbol{v}}_{i}^{\prime}\right]_{x^{\prime} y^{\prime}}\right\|^{2}
\end{gathered}
$$

where $n$ denotes the number of pairs of corresponding points extracted from the two images and $\hat{\boldsymbol{P}}$ and $\hat{\boldsymbol{P}}^{\prime}$ denote the sets of $3 \mathrm{D}$ position vectors of bifurcation points $\hat{\boldsymbol{p}}_{i}=\left(\hat{x}_{i}, \hat{y}_{i}, \hat{z}_{i}\right)$ and $\hat{\boldsymbol{p}}^{\prime}{ }_{i}=\left(\hat{x}_{i}^{\prime}, \hat{y}_{i}^{\prime}, \hat{z}_{i}^{\prime}\right)$, respectively, $\hat{\boldsymbol{\nu}}_{i}$ and $\hat{\boldsymbol{\nu}}_{i}^{\prime}$, denote the respective $2 \mathrm{D}$ vessel directional vectors of bifurcations in each views, and $\hat{\boldsymbol{v}}=\left\{\hat{\boldsymbol{v}}_{1}, \hat{\boldsymbol{v}}_{2}, \ldots, \hat{\boldsymbol{v}}_{n}\right\}$ and $\hat{\boldsymbol{v}}^{\prime}=\left\{\hat{\boldsymbol{v}}_{1}^{\prime}, \hat{\boldsymbol{v}}_{2}{ }_{2}, \ldots, \hat{\boldsymbol{v}}_{n}^{\prime}\right\}$ denote the calculated $3 \mathrm{D}$ vessel directional vectors of bifurcations in two views, and $\left[\hat{\boldsymbol{v}}_{i}\right]_{x y}$ and $\left[\hat{\boldsymbol{v}}^{\prime}{ }_{i}\right]_{x^{\prime} y^{\prime}}, i=$ $1, \ldots, n$, denote the calculated $2 \mathrm{D}$ projection vector at bifurcation on the image planes, $c_{\boldsymbol{k}}$ denotes the respective $k$ th column vectors of matrix $\boldsymbol{R}$, and $\boldsymbol{R}^{-1}$ is the inverse matrix of $\boldsymbol{R}$.

\subsection{Calculation of 3D coronary arterial tree}

After the transformation $(\boldsymbol{R}, \boldsymbol{t})$ that defines the two views is obtained, this information will be used for establishing the point correspondences on $2 \mathrm{D}$ vessel centerlines in the pair of images and calculating 3D morphologic structures of coronary arterial tree. The calculated transformation in conjunction with the epi-polar constraints were employed as the framework for establishing the point correspondences on the vessel centerlines based on the two identified 2D coronary arterial trees [24]. Since the information of relative orientations and locations of two cameras is known (i.e., in term of a transformation calculated previously), the correspondences of image points can be solved by employing "epi-polar constraints" theory [26].

With the point correspondences on $2 \mathrm{D}$ vessel centerlines $\left(\xi_{j}, \eta_{j}\right)$ and $\left(\xi_{j}^{\prime}, \eta_{j}^{\prime}\right)$ and the transformation $(\boldsymbol{R}, \boldsymbol{t})$, the $3 \mathrm{D}$ vessel centerline points of coronary arteries $\left(x_{j}, y_{j}, z_{j}\right)$ can then be calculated [24][25]. To recover the morphology of the 3D arterial lumen, the contour points on each circular lumen cross section centered at $\left(x_{i}, y_{i}, z_{i}\right)$ are calculated. The normal vector at the plane spanned by the cross section is parallel to the tangent vector at point $\left(x_{i}, y_{i}, z_{i}\right)$ on the vessel skeleton (or $3 \mathrm{D}$ vessel centerline). For any cross section of vessel segment, it can be modeled as a circular disk centered at $\left(x_{i}, y_{i}, z_{i}\right)$ with diameter $d_{i}$. Then the $3 \mathrm{D}$ lumen surface is easily represented by filling up the polygons between every two consecutive circular disks.

\section{Determination of Optimal Views}

The reconstructed $3 \mathrm{D}$ coronary arterial tree can be rotated to any selected viewing angle yielding multiple computer-generated projections to determine for each patient which standard views are useful and which are of no clinical value due to excessive overlap. Therefore, the $3 \mathrm{D}$ computer assistance provides means to improve the quality and utility of the images subsequently acquired. When an 
arbitrary computer-generated image is produced, the gantry information defining the current projection is calculated in the form of left anterior oblique/right anterior oblique (LAO/RAO) and caudal/cranial (CAUD/CRAN). These gantry angles are defined in a spatial coordinate system with the iso-center as the origin. The LAO/RAO angulation is defined on the $y$ - $z$ plane, while the CAUD/CRAN angulation is defined on the $x$ - $z$ plane. With the spatial coordinates, the position of focal spot $\left(x_{f}, y_{f}, z_{f}\right)$ can be formulated by use of two rotations $R_{x}(\alpha)$ and $R_{y}(-\beta)$ as,

$$
\left[x_{f}, y_{f}, z_{f}\right]=\left[x_{n}, y_{n}, z_{n}\right] \cdot R_{x}(\alpha) R_{y}(-\beta)
$$

where $\left(x_{n}, y_{n}, z_{n}\right)=\left(0,0, D_{f c}\right)$ denotes the neutral position of focal spot (or anterior-posterior (AP) view with $0^{\circ} \mathrm{LAO}$ and $0^{\circ} \mathrm{CAUD}, D_{f c}$ is the distance between iso-center and focal spot (which is provided by manufacture), $R_{x}$ and $R_{y}$ denotes the rigid rotations with respect to $x$-axis and $y$-axis, and $\alpha$ and $\beta$ denote the LAO and CAUD angles, respectively.

Let $p_{i}, i=0,1, \ldots, m$ denote the points along the centerline of a $3 \mathrm{D}$ vessel $l$. Let $\boldsymbol{l}_{j}=\left[l_{j_{x}}, l_{j_{y}}, l_{j_{z}}\right]^{t}, j=1,2, \ldots, m$ denote the vectors associated with individual sections between $p_{j-1}$ and $p_{j}$. The functions $\mathcal{S}(\alpha, \beta, l)$ and $\mathcal{O}(\alpha, \beta, l)$ in term of gantry orientation ( $\alpha$ and $\beta$ angles) for evaluation of degrees of foreshortening and overlap with respect to the selected arterial segment $l$ can be defined as follows:

$$
\begin{gathered}
\mathcal{S}(\alpha, \beta, l)=\sum_{j=1}^{m}\left\|\boldsymbol{l}_{j} \cos \left(\theta_{j}\right)\right\|^{2}=\sum_{j=1}^{m}\left(\boldsymbol{l}_{j} \cdot \boldsymbol{z}_{p}\right)^{2} \\
\mathcal{O}(\alpha, \beta, l)=\mathcal{N}\left(\left\{\sum_{i=1}^{n} \sum_{j=1}^{m_{i}} \Pi\left(\hat{l}_{j}^{i}\right)\right\} \bigcap\left\{\sum_{k=1}^{m} \Pi\left(\boldsymbol{l}_{k}\right)\right\}\right) / \mathcal{N}\left(\sum_{j=1}^{m} \Pi\left(\boldsymbol{l}_{j}\right)\right)
\end{gathered}
$$

subject to the constraints pertinent to the achievable gantry angles of imaging system (which depend on different manufacture designs)

$$
-120^{\circ}<\alpha<120^{\circ}, \quad-60^{\circ}<\beta<60^{\circ},
$$

where "." denotes the inner product and $\theta_{j}$ is the angle between the directional vector $l_{j}$ and projection vector $z_{p}=[-\cos (\alpha) \sin (\beta),-\sin (\alpha), \cos (\alpha) \cos (\beta)]^{t}$, and where $\hat{l}_{j}^{i}$ denotes the $j$-th segment of the $i$-th artery (consisting of $m_{i}$ segments) in the coronary tree (consisting of $n$ coronary arteries), $\Pi(\cdot)$ denotes the operator projecting an artery segment onto the image plane in the forms of a pixel-based array, $\bigcap$ denotes the intersection operator, and $\mathcal{N}(\cdot)$ is the function that counts the number of pixels onto the pixel-based array. On the basis of Eqs. (3) and (4), the composite function $\mathcal{V}(\alpha, \beta, l)=\mathcal{S}(\alpha, \beta, l) \oplus \mathcal{O}(\alpha, \beta, l)$ can be derived which incorporates the measurements of foreshortening and overlap by a fusion operator $\oplus$. Based on the function $\mathcal{V}(\alpha, \beta, l)$, a set of gantry angles $(\tilde{\alpha}, \tilde{\beta})$ 's which minimize the vessel foreshortening and overlap relative to the artery $l$ can be determined and utilized to acquire subsequent views for diagnostic or therapeutic procedure during coronary intervention. 


\section{Experimental Results}

The accuracy of the proposed method was evaluated by use of 8 pairs of angiograms containing a Johnson \& Johnson catheter guide wire with markers of $15 \mathrm{~mm}$ inter-distance. Images were acquired from Philips Integris H 3000 at various viewing angles. Eight markers (in terms of $2 \mathrm{D}$ points) along with the wire (in term of a $2 \mathrm{D}$ curve) were first identified manually on both images. With the identified marker points, the transformation that characterized the two views was calculated. Afterwards, the 3D catheter guide wire was reconstructed by use of the proposed technique. The length of catheter wire was calculated resulting in root-mean-square $3.1 \%$ error relative to the actual length $105 \mathrm{~mm}$.

The total processing time varies from 7 to 10 minutes including image transfer ( 3 - 4 minutes) and all the computational processing ( $4-6$ minutes). Angiograms of more than 200 cases acquired from a single-plane or biplane system have been completed for $3 \mathrm{D}$ reconstruction. The transformation was determined without the need of calibration object, and the 3D coronary arterial trees were reconstructed including left coronary, right coronary, and bypass graft systems. Among these studies, one example was chosen for demonstration in this paper. In Figs. 1(a) and 1(b), a pair of angiograms of LCA tree acquired from a biplane imaging system are shown. The reconstructed $3 \mathrm{D}$ left coronary arterial tree was obtained and viewed at gantry angulation $31.6^{\circ}$ LAO and $49.3^{\circ}$ CAUD after the process of 3D reconstruction was employed where the arterial segment $(9.8$ $\mathrm{mm}$ ) of interest near the left coronary circumflex artery was manually marked as shown in Fig. 1(c).

With the selected arterial segment (i.e., corresponding to a lesion), the process of optimal strategy was applied resulting in the estimates of foreshortening, overlap, and composite maps as shown in 1(d), 1(e), and 1(f). Each map consists of four quadrants characterizing the gantry orientations in terms of (LAO, CRAN), (RAO, CRAN), (RAO, CAUD), and (LAO, CAUD), respectively. Due to the mechanical limitation, the moving range of the gantry arm is confined from $120^{\circ} \mathrm{LAO}$ to $120^{\circ} \mathrm{RAO}$ and from $60^{\circ}$ CRAN to $60^{\circ} \mathrm{CAUD}$. The degrees of foreshortening and overlap in the respective maps are characterized by 5 different regions with individual shaded gray levels. The composite map is defined based on a fusion scheme such that the measures of foreshortening serve as the basis superimposed with the measures of overlap.

By use of the maps as shown in Figs. 1(d)-(f), any computer-predicted projection can be "previewed" accompanied with the estimates of foreshortening and overlap by pointing at any location in the map. Figs. 2(a)-(c) show three typical examples of unappreciated views. The remaining two views as shown in Figs. 2(d) and (e) demonstrate no vessel overlap and $0 \%$ foreshortening with respect to the selected arterial segment of interest, which can be utilized for acquiring subsequent images to facilitate further clinical diagnosis. 


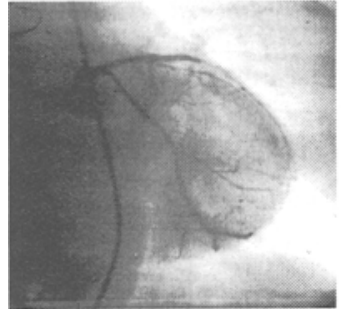

(a)

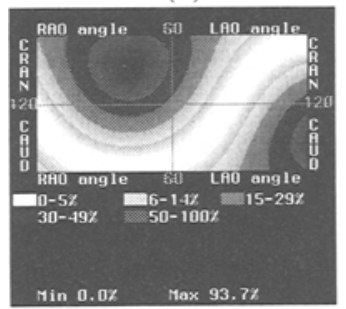

(d)

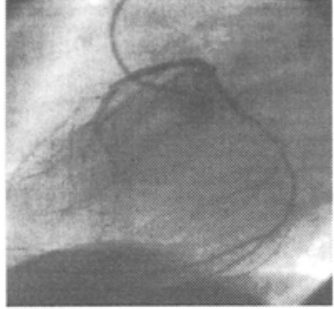

(b)

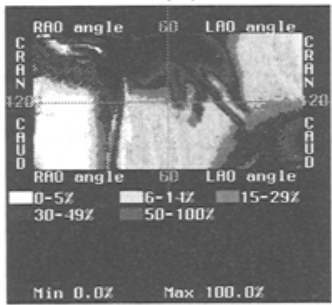

(e)

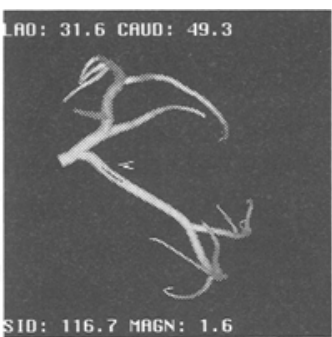

(c)

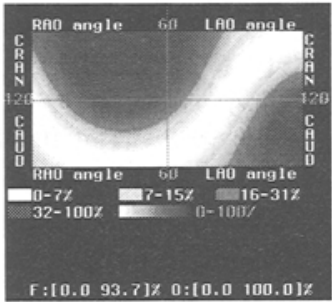

(f)

Fig. 1. (a)(b) The employed first $\left(0^{\circ} \mathrm{LAO}, 16^{\circ} \mathrm{CAUD}\right)$ and second views $\left(89^{\circ} \mathrm{LAO}\right.$, $1^{\circ}$ CAUD) of left coronary artery angiograms. (c) The selected segment of interest (9.8 $\mathrm{mm}$ ) with $17 \%$ foreshortening based on gantry angulation ( $31.6^{\circ} \mathrm{LAO}, 49.3^{\circ}$ CAUD). The foreshortening map (d), the overlap map (e), and the composite map (f) resulting from (d) and (e) illustrate various degrees of respective overlap, foreshortening, and combination relative to the selected arterial segment.

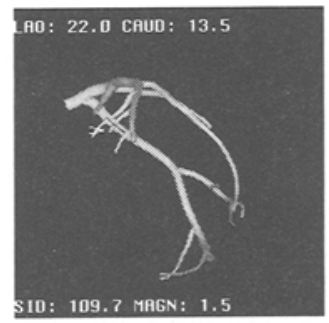

(a)

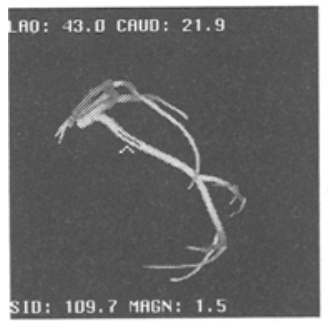

(d)

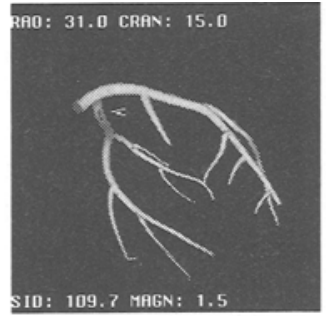

(b)

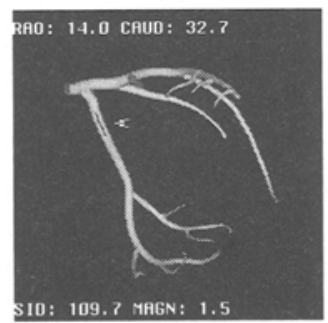

(e)

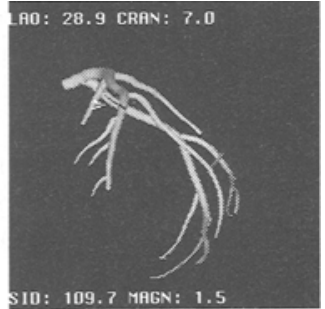

(c)

Fig. 2. Unappreciated views: (a) $0 \%$ foreshortening and $27 \%$ overlap, (b) $45 \%$ foreshortening and no overlap, and (c) $18 \%$ foreshortening and $40 \%$ overlap. (d)(e) Views with $0 \%$ foreshortening and no vessel overlap adequate to be utilized for subsequent image acquisitions. 


\section{Concluding Remarks}

In this paper, a novel method is proposed for on-line reconstruction 3D coronary arterial tree from routine angiograms acquired at arbitrary angles and without using calibration objects based on a single-plane or biplane imaging system. More than 120 cases of coronary arterial systems have been reconstructed including LCA, RCA, and bypass grafts. A validation confirmed the accuracy of 3D length measurement to within RMS $3.1 \%$ error using 6 pairs of angiograms of catheter guide wire. The length of employed wire is $105 \mathrm{~mm}$ with 8 markers of $15 \mathrm{~mm}$ inter-distance. The choice of an optimal view of the vasculature of interest can be achieved on the basis of the capability of rotating the reconstructed 3D coronary arterial tree with the derived foreshortening, overlap, and composite maps. Such a unique capability thus provides an optimal visualization strategy that should lead to more efficient and successful diagnostic and therapeutic procedures in assessment of lesion length and diameter narrowing.

\section{Acknowledgment}

This project is supported by the Whitaker Foundation. The help of Dr. Bertron M. Groves in providing some angiograms and Keith E. Hellman in providing graphical user interface for image transfer is greatly appreciated.

\section{References}

1. H.C. Kim, B.G. Min, T.S. Lee, et. al., "3D digital subtraction angiography," IEEE Trans. Med. Imag., vol. MI-1, pp. 152-158, 1982.

2. K.L. Parker, K.L. Pope, R. van Bree, et. al., "3-d reconstruction of moving arterial beds from digital subtraction angiography," Comput. Biomed. Res., vol. 20, pp. $166-185,1987$.

3. K. Kitamura, J.M. Tobis, and J. Sklansky, "Estimating the 3D skeletons and transverse areas of coronary arteries from biplane angiograms," IEEE Trans. on MI, vol. MI-7, pp. 173-187, 1988.

4. T. Saito, M. Misaki, K. Shirato, and T. Takishima, "Three-dimensional quantitative coronary angiography," IEEE Trans. on Bio. Eng., vol. 37, no. 8, pp. 768-777, Aug. 1990.

5. C.P. Pellot, A. Herment, M. Sigelle, P. Horain, H. Maitre, and P. Peronneau, "A 3d reconstruction of vascular structures from two $x$-ray angiograms using an adapted simulated annealing algorithm," IEEE Tran. on Med. Imag., vol. 13, no. 1., pp. 49-60, Mar. 1994.

6. N. Guggenheim, P.A. Doriot, et al., "Spatial reconstruction of coronary arteries from angiographic images," Phys. in Medic. E Biol., vol. 36, pp. 99-100, 1991.

7. A. Wahle, E. Wellnhofer, I. Mugaragu, H.U. Sauer, H. Oswald, and E. Fleck, "Assessment of diffuse coronary artery disease by quantitative analysis of coronary morphology based upon 3-d reconstruction from biplane angiograms," IEEE Trans. on $M I$, vol. 14, no. 2, pp. 230-241, 1995.

8. S. Stansfield, "ANGI: A rule based expert system for automatic segmentation of coronary vessels from digital subtracted angiograms," IEEE Trans. on PAMI, vol. 8, no. 2, pp. 188-199, 1986. 
9. C. Smets, F. Vandewerf, P. Suetens, and A. Oosterlinck, "An expert system for the labeling and $3 \mathrm{~d}$ reconstruction of the coronary arteries from two projections," Int. J. Card. Img., vol. 5, no. 2-3, pp. 145-154, 1990.

10. G. Coppini, M. Demi, R. Mennini, G. Valli, "3D knowledge driven reconstruction of coronary trees," Medical \& Bio. Eng. \& Comp., pp. 535-542, 1991.

11. D. Delaere, C. Smets, P. Suetens, G. Marchal, and F. Van de Werf, "Knowledgebased system for the $3 \mathrm{~d}$ reconstruction of blood vessels from two angiographic projections," Med. Biol. Eng. Comput, vol. 29, no. 6, pp. ns27-ns36, Nov. 1991.

12. I. Liu and Y. Sun, "Fully automated reconstruction of 3-d vascular tree structures from two orthogonal views using computational algorithms and production rules", Optical Engineering, vol. 31, no. 10, pp. 2197-2207, Oct. 1992.

13. A. Rouge, C. Picard, D. Sanit-Felix, et al., "3-d coronary arteriography," Int. J. of Card. Img., vol. 10, pp. 67-70, 1994.

14. H. C. Longuet-Higgins, "A computer algorithm for reconstructing a scene from two projections," Nature, vol. 293, no. 10, pp. 133-135, September 1981.

15. R. Y. Tsai and T. S. Huang, "Uniqueness and estimation of 3D motion parameters of rigid objects with curved surfaces," IEEE Trans. on PAMI, vol. 6, no. 1, pp. 13-27, Jan. 1984.

16. J.Q. Fang, T.S. Huang, "Some experiments on estimating the 3-d motion parameters of a rigid body from two consecutive image frames," IEEE Trans. on PAMI, vol. 6, pp. 547-554, Jan. 1984.

17. J. Philip, "Estimation of 3-d motion of rigid objects from noisy observations," IEEE Trans. on PAMI, vol. 13, pp. 61-66, 1991.

18. C. E. Metz and L. E. Fencil, "Determination of three-dimensional structure in biplane radiography without prior knowledge of the relationship between the two views: Theory," Medical Physics, 16 (1), pp. 45-51, Jan/Feb 1989.

19. L. E. Fencil and C. E. Metz, "Propagation and reduction of error in threedimensional structure determined from biplane views of unknown orientation," Medical Physics, 17 (6), pp. 951-961, Nov/Dec 1990.

20. J. Weng, T.S. Huang, and N. Ahuja, "A two-step approach to optimal motion and structure estimation," Proc. IEEE Workshop Computer Vision, pp. 355-357, 1987.

21. J. Weng, N. Ahuja, and T. Huang, "Closed-form solution and maximum likelihood: a robust approach to motion and structure estimation," Proc. IEEE Conf. Computer Vision and Pattern Recognition, pp. 381-386, 1988.

22. J. Weng, T.S Huang, N. Ahuja, "Motion and structure from two perspective view: algorithms, error analysis and error estimation," IEEE Trans. on PAMI, vol. 11, pp. 451-476, Jan. 1989.

23. J. Weng, N. Ahuja, T.S. Huang, "Optimal motion and structure estimation," IEEE Trans. on PAMI, vol. 15, no. 9, pp. 864-884, 1993.

24. S.-Y. J. Chen, K.R. Hoffmann, J.D. Carroll, "Three-dimensional reconstruction of coronary arterial tree based on biplane angiograms," Proceedings of SPIE Medical Imaging: Image Processing, vol. 2710, Newport Beach, California, pp. 103-114, 1996

25. S.-Y. J. Chen and C.E. Metz, "Improved determination of biplane imaging geometry from two projection images and its application to 3D reconstruction of coronary arterial trees," Medical Physics, vol. 24, no. 5, May 1997, pp. 633-654.

26. B.K.P. Horn, Robot Vision, The MIT Press, McGraw-Hill Book Company, 1986. 